\title{
LISTA ANOTADA DE LOS ESCORPIONES (ARACHNIDA: SCORPIONES) DE LA ESPAÑOLA (REPÚBLICA DOMINICANA Y HAITÍ)
}

\author{
Gabriel de los Santos ${ }^{1}$, Luis F. de Armas $^{2}$ y Rolando Teruel ${ }^{3}$
}

${ }^{1}$ Museo Nacional de Historia Natural "Prof. Eugenio de Jesús Marcano".

Calle César Nicolás Penson, Plaza de la Cultura Juan Pablo Duarte, Santo Domingo, 10204, República Dominicana. g.delossantos@mnhn.gov.do

${ }^{2}$ Apartado Postal 4327, San Antonio de los Baños, Artemisa 32500, Cuba. luisdearmas1945@gmail.com

${ }^{3}$ Centro Oriental de Ecosistemas y Biodiversidad (Bioeco), Museo de Historia Natural "Tomás Romay". José A. Saco \# 601, esquina Barnada, Santiago de Cuba 90100, Cuba. rteruel@bioeco.cu

\section{RESUMEN}

Se listan los escorpiones de La Española (República Dominicana y Haití) conocidos hasta ahora: tres familias (Buthidae, Hormuridae y Scorpionidae), ocho géneros, 46 especies vivientes y cuatro especies fósiles. Buthidae, con cinco géneros y 37 especies vivientes, es la familia más diversificada en esta isla antillana, siendo Tityus C. L. Koch, 1836, Microtityus Kjellesvig-Waering, 1966 y Centruroides Marx, 1890, los géneros de mayor distribución geográfica y riqueza específica con 14, 12 y siete especies, respectivamente. República Dominicana (que ocupa las dos terceras partes orientales de la isla) posee 45 especies, 36 de ellas endémicas del país, mientras que Haití cuenta con nueve especies que incluyen un solo endemismo nacional. El único taxon no endémico de la isla es Isometrus maculatus (De Geer, 1778), introducido en ambos países. Esta es la única isla antillana que contiene representantes tanto de la familia Hormuridae como de la fauna fósil (en ámbar).

Palabras clave: Scorpiones, Buthidae, Hormuridae, Scorpionidae, taxonomía, La Española, Antillas, Región Neotropical.

Title: Annotated list of the scorpions (Arachnida: Scorpiones) of Hispaniola (Dominican Republic and Haiti).

\section{ABSTRACT}

It is given an annotated list of the known scorpions of Hispaniola (Dominican Republic and Haiti), which belong to three families (Buthidae, Hormuridae, Scorpionidae), eight genera and 46 recent and four fossil species. Buthidae (five genera and 37 living species) is the most diversified family in this Antillean island, being Tityus C. L. Koch, 1836, Microtityus Kjellesvig-Waering, 1966, and Centruroides Marx, 1890, the most diverse and widespread genera with 14, 12 and seven species, respectively. The Dominican Republic (that represents the eastern two-thirds of the island), has 45 species and 36 of them are endemic from the country, whereas Haiti accounts nine species with a single national endemism. Isometrus maculatus (De Geer, 1778) has been introduced in both countries and is the only non-endemic species. This is the only Antillean island which includes representatives of both the Hormuridae family and the fossil fauna (in amber).

Keywords: Scorpiones, Buthidae, Hormuridae, Scorpionidae, taxonomy, Hispaniola, Antilles, Neotropical Region. 


\section{INTRODUCCIÓN}

Entre las islas que baña el mar Caribe, La Española (o Hispaniola, como también se le conoce), con $76480 \mathrm{~km}^{2}$, es la segunda en extensión y conjuntamente con Cuba, Jamaica y Puerto Rico integra las Antillas Mayores. Su territorio está políticamente compartido por dos países: la República de Haití en el oeste y la República Dominicana, que ocupa las dos terceras partes orientales. La isla es mayormente montañosa y está surcada del noroeste al sudeste por varias cordilleras paralelas y mayormente aisladas entre sí: la más extensa de ellas es la Cordillera Central, cuya máxima elevación (3 $175 \mathrm{msnm}$ ) constituye la más importante de todo el archipiélago antillano. Gran parte de la vertiente meridional y ciertas áreas de la septentrional están cubiertas por vegetación xerófita o semixerófita.

La historia de esta isla recoge constantes cambios, tanto en su nombre como en el territorio ocupado por los estados que la integran, dando origen a confusiones e incertidumbre respecto a la verdadera localidad de algunos táxones descritos o registrados de sus predios. Los aborígenes de ascendencia arahuaca nombraban de manera diferente diversas regiones de la isla, siendo Haití uno de esos nombres. En diciembre de 1492 Cristóbal Colón la bautizó La Española (nombre que el italiano Pedro Mártir de Anglería latinizaría como Hispaniola) y en 1697, por el Tratado de Rijswijk (o Ryswick) España cedió a Francia la porción occidental de la isla, la que pasó a nombrarse Saint Domingue; la otra parte continuó en su poder y fue conocida como Santo Domingo. Casi dos siglos más tarde, los eventos políticos que tuvieron lugar durante la primera mitad del siglo XIX derivaron en las declaraciones de independencia de las dos repúblicas que hoy comparten la isla. Por esto, los especímenes colectados antes del siglo XX han sido mencionados indistintamente como procedentes de Haití, La Española, Hispaniola, Saint-Domingue, Santo Domingo y otros nombres usados. Por estas consideraciones, entendemos que la mejor manera de esclarecer su origen es analizar cuidadosamente la fecha de captura, etiquetado y/o publicación de los especímenes.

Palisot de Beauvois (Ambroise Marie François Joseph Palisot, barón de Beauvois, 1752-1820) arribó en 1778 a Haití, donde vivió varios años, durante los cuales recolectó insectos y arácnidos (Wetherbee, 1989). En 1805 comenzó a publicar los resultados de sus investigaciones, las que con su muerte quedaron inconclusas. Entre los alacranes que mencionó de esta isla se halla Scorpio americanus [según Fet y Lowe (2000: 282), una grafía incorrecta de Scorpio americus Linnaeus, 1758 y un error de identificación, posiblemente referido a Centruroides nitidus (Thorell, 1876)], en tanto Scorpio lepturus [= Opisthacanthus lepturus (Palisot de Beauvois, 1805)] fue erróneamente descrita como de Sudamérica. Durante mucho tiempo se creyó que esta era la misma que habita en Panamá y Colombia, pero Armas y Marcano Fondeur (1992), al comparar ejemplares de Panamá con los de República Dominicana, concluyeron que ambas poblaciones diferían taxonómicamente y que los especímenes dominicanos eran similares a los tipos de Opisthacanthus kinbergii laevicauda Thorell, 1876, descrita de una localidad desconocida, por lo que la elevaron al nivel de especie y la consideraron endémica de La Española . Lourenço (1995), examinó la antigua colección de alacranes de Eugène Simon, depositada en el Museo Nacional de Historia Natural (París) y localizó lo que pudiera ser el tipo de O. lepturus: un macho adulto (RS-0305) recolectado en "Saint-Domingue" [posiblemente Haití en vez de República Dominicana, como erróneamente citó Fet (2000: 402)]. Ciertamente, este ejemplar no es asignable a $O$. elatus pero es similar a $O$. kinbergii laevicauda, por lo que Lourenço (1995) reconoció la validez de O. lepturus (cuya distribución restringió a La Española) y de $O$. elatus e incluyó a $O$. kinbergii laevicauda en la sinonimia de la primera.

Thorell (1876) describió de esta isla cinco especies de Buthidae: Centrurus nitidus, Centrurus tenuis, Centrurus republicanus, Isometrus antillanus e Isometrus crassimanus. 
Las tres primeras fueron consideradas por Kraepelin (1891) como una sola [actualmente C. nitidus], en tanto que las dos últimas permanecieron muy mal conocidas hasta que Francke y Santiago-Blay (1984) establecieron que se trataba de una sola especie [Tityus crassimanus] que habita en La Española (I. crassimanus había sido erróneamente descrita de México e I. antillanus de las Antillas). Thorell indicó que los ejemplares de $C$. nitidus procedían de "América (India Occidentalis)" y de Nueva York (esta última, un error evidente). Por otra parte, Pocock (1902) consignó que los ejemplares examinados por Thorell procedían de Haití [Fet y Lowe (2000: 115) citaron erróneamente la localidad tipo como "Santo Domingo, República Dominicana”].

En el caso de C. republicanus (la serie tipo procede de Puerto Príncipe, Haití), la sinonimia propuesta por Kraepelin (1891) ha sido aceptada sin discrepancias. Sin embargo, la posible validez de Centruroides tenuis fue sugerida por Armas y Marcano Fondeur (1987) y Armas (2001a). Según Thorell (1876), los tipos de esta especie proceden de "Saint Domingue” (¿Haití?) y de «New York».

Karsch (1879) describió Centrurus princeps [=Rhopalurus princeps (Karsch, 1879)], a partir de un macho recolectado por Carl Ehrenburg en Puerto Príncipe, Haití. Según Wetherbee (1989), Ehrenburg (1801-1849) estuvo recolectando plantas en este país entre 1828 y 1831.

Pocock (1893), en lo que sin lugar a dudas constituyó la más importante revisión del siglo XIX sobre los alacranes antillanos, mencionó siete especies para "Saint-Domingue" o Haití, indistintamente. Tres de ellas [Tityus americanus (Linnaeus, 1758), Tityus obtusus (Karsch, 1879) y Heteroctenus junceus (Herbst, 1800), esta última ahora en el género Rhopalurus Thorell, 1876] no forman parte de la fauna de esta isla. La primera constituye un sinónimo posterior de Scorpio americus Linnaeus, que es una especie incertae sedis (Fet y Lowe, 2000: 282). La segunda representa un error de identificación de Tityus crassimanus de acuerdo con Francke y Santiago-Blay (1984), en tanto que la tercera constituye un error de identificación de alguna de las tres especies autóctonas de Rhopalurus (véanse: Armas, 1981c, 1982a, 1988a; Teruel y Armas, 2012). En esta contribución, Pocock también mencionó la presencia en la isla de Isometrus maculatus (DeGeer, 1778), un alacrán introducido en las Antillas y gran parte del mundo.

Pocock (1898) describió Diplocentrus politus (actualmente en el género Cazierius Francke, 1978), a partir de dos hembras y un macho inmaduro supuestamente recolectados en Brasil, pero Armas (1981a) demostró que es propia de La Española, cuestión que Francke (1978) ya había sugerido sobre la base de evidencias biogeográficas.

Kraepelin (1901) mencionó cuatro especies para "Saint-Domingue" o Haití, de modo indistinto. Tres de ellas ya habían sido referidas por Pocock (1893), pero Diplocentrus gundlachii Karsch, 1880 constituye un error de identificación. Por otra parte, Roewer (1943: 219) citó Centruroides testaceus (De Geer, 1778) de Haití, Centruroides vittatus (Say, 1821) de "Saint-Domingue" y Centruroides nitidus de Texas, EE.UU. Estos tres registros constituyen errores de identificación o contienen un error en la localidad consignada; desafortunadamente, todo el material de referencia se perdió en el correo antes de ser examinado (L. F. de Armas, datos inéditos).

Durante el último cuarto del siglo XX, el estudio de los alacranes de La Española (mayormente de República Dominicana) cobró un inusitado auge. Armas (1976a) mencionó del Lago Enriquillo, provincia de Independencia, como Centruroides nitidus, lo que poco después fue descrito como C. bani Armas y Marcano Fondeur, 1987, constituyendo esta la primera referencia precisa a un alacrán de este país. 
Las contribuciones de Armas (1981a-b, 1982b, 1987, 1999, 2001a-b, 2002a-b, 2005), Santiago-Blay (1985), Armas y Marcano Fondeur (1987, 1988, 1992), Armas y Abud Antun (1992, 2000, 2004, 2015), Lourenço (1995), Lourenço et al. (1999, 2000), Rouaud et al. (2002), Armas y Prieto Trueba (2003), Teruel (2005), Prendini et al. (2009); Armas y Teruel (2012, 2016), Kovař́k y Teruel (2014), Lourenço y Armas (2015) y Teruel et al. (2015a-b), incrementaron de modo sustancial el conocimiento sobre la taxonomía, biología y biogeografía de la escorpiofauna de La Española.

Santiago-Blay (1990) presentó su tesis doctoral sobre los alacranes de La Española, pero lamentablemente nunca publicó los nuevos táxones contenidos en dicho documento, muchos de los cuales posiblemente hayan sido descritos posteriormente en las contribuciones citadas en el párrafo previo.

Rudloff(1994a-b) publicó parte de su estudio sobre los escorpiones de las Antillas, donde se incluyen muchos de los taxones dominicanos, pero en gran medida dicha obra es una adaptación al alemán de los trabajos de Armas (1986, 1988a).

Por otra parte, hasta el momento se han identificado cuatro géneros de alacranes fósiles de La Española: tres de Buthidae (Centruroides, Microtityus, Tityus) y uno de Scorpionidae (Heteronebo), todos ellos contenidos en ámbar dominicano (Schawaller, 1979, 1982; Armas, 1988b; Santiago-Blay y Poinar, 1988; Santiago-Blay et al., 1990; Pérez-Gelabert, 1999; Lourenço, 2009 y 2013). La especie de Centruroides fue relegada por Armas (1988a) como un sinónimo más moderno de $C$. nitidus, en tanto la de Scorpionidae solamente fue identificada a nivel de género (Santiago-Blay y Craig, 1998).

En la actualidad, los escorpiones se sitúan como uno de los grupos de Arachnida mejor estudiados en La Española, aunque continúan siendo objeto de investigación. La cifra total de especies vivientes hasta ahora descritas o registradas de esta isla asciende a 46, repartidas en ocho géneros y tres familias, siendo la única isla antillana en la que está representada la familia Hormuridae, así como la única en la que se han hallado especies fósiles.

Respecto a otras islas antillanas, la escorpiofauna de La Española es la segunda en importancia después de Cuba. Sin embargo, debido a la intensa deforestación a que fue sometido el territorio haitiano, la inmensa mayoría de esta fauna se localiza en la parte dominicana, cuyos ecosistemas están mejor conservados.

Durante los últimos 30 años se han publicado varias listas y catálogos que abordan directamente la escorpiofauna de La Española (Teruel, 2005; Perez-Gelabert, 2008) o incluyen información sobre la misma (Armas, 1988a; Fet et al., 2000; Armas, 2001a). Con independencia de los posibles errores que cada una de estas contribuciones contenga, todas han quedado definitivamente desactualizadas debido al cúmulo de información publicada durante los últimos 15 años.

\section{OBJETIVOS}

- Elaborar una lista actualizada de las especies de escorpiones de La Española.

- Dar a conocer los principales hitos en el estudio de los escorpiones de La Española, aclarando confusiones y corrigiendo yerros que aparecen en la literatura sobre este grupo. 


\section{MATERIALES Y MÉTODOS}

Para la clasificación supragenérica se siguen los criterios de Fet y Soleglad (2005), excepto para Hormuridae, taxon para el que se aceptó la propuesta de Prendini y Wheeler (2005) de tratarlo como una familia independiente. La información se considera actualizada hasta el 30 de junio de 2016.

Para cada especie se incluyen únicamente las referencias de mayor relevancia: catálogos generales, revisiones y aquellas en las que se aporta información sustancial, ya sea taxonómica o biológica. Los mapas de distribución representan una compilación actualizada y corregida a partir de dos fuentes independientes: 1) las referencias publicadas, cuya veracidad ha sido comprobada por los presentes autores; 2) los datos aún inéditos, recopilados por los presentes autores a partir de su propio trabajo de campo y/o de los especímenes de las colecciones bajo su custodia y fotografías digitales de alta resolución facilitados por colegas confiables. Todas las fotografías de escorpiones aquí incluidas corresponden a individuos adultos y a menos que se indique lo contrario, fueron tomadas in situ por uno de los autores (RT), mediante una cámara digital Nikon Coolpix S-8100 y modificadas mínimamente con Adobe Photoshop CS5 (únicamente ajuste de brillo, contraste y otros parámetros de impresión).

Simbología empleada. La distribución geográfica conocida de cada especie está simbolizada como: RD (presente en la República Dominicana) y H (presente en la República de Haití). En la "Lista anotada", los géneros y especies válidos aparecen en negritas la primera vez que son citados. $(\dagger)=$ especie fósil.

\section{RESULTADOS}

Las 46 especies vivientes y las cuatro fósiles hasta ahora descritas o registradas de La Española (Tabla I), pertenecen a ocho géneros y tres familias: Buthidae, Hormuridae y Scorpionidae.

Tabla I. Composición taxonómica y endemismo (entre paréntesis) de los escorpiones de La Española y sus dos países integrantes. Notas: a) sólo se incluyen los táxones nominales; b) el valor de endemismo es relativo para cada país y absoluto para la isla.

\begin{tabular}{cccc}
\hline Género & $\begin{array}{c}\text { Haití } \\
\text { No. de especies }\end{array}$ & $\begin{array}{c}\text { República Dominicana } \\
\text { No. de especies }\end{array}$ & $\begin{array}{c}\text { La Española } \\
\text { No. de especies }\end{array}$ \\
\hline Opisthacanthus & $1(0)$ & $1(0)$ & $1(1)$ \\
Cazierius & $1(0)$ & $3(2)$ & $3(3)$ \\
Heteronebo & $2(1)$ & $4(3)$ & $5(5)$ \\
Centruroides & $3(0)$ & $7(4)$ & $7(7)$ \\
Isometrus & $1(0)$ & $1(0)$ & $1(0)$ \\
Microtityus & $0(0)$ & $12(12)$ & $12(12)$ \\
Rhopalurus & $1(0)$ & $3(2)$ & $3(3)$ \\
Tityus & $1(0)$ & $14(13)$ & $14(14)$ \\
\hline Totales & $9(1)$ & $45(36)$ & $46(45)$ \\
\hline
\end{tabular}


Buthidae, con cinco géneros y 37 especies, es la más diversificada en esta isla antillana, siendo Tityus C. L. Koch, 1836, Microtityus Kjellesvig-Waering, 1966 y Centruroides Marx, 1890, los géneros de mayor distribución geográfica y riqueza con 14, 12 y siete especies, respectivamente. Con tres especies descritas, Tityus es también el género con mayor cantidad de especies fósiles en esta isla.

La República Dominicana posee 45 especies, de las cuales 36 son endémicas de dicho país. Por su parte, Haití cuenta con nueve especies, incluyendo un solo endemismo nacional. El único taxon no endémico de la isla es Isometrus maculatus (De Geer, 1778), introducido en ambos países.

\section{LISTA ANOTADA DE LAS ESPECIES DE ESCORPIONES DE LA ESPAÑOLA}

\section{Familia Buthidae C. L. Koch, 1837}

Género Centruroides Marx, 1890. (Fig. 3 a).

Centruroides alayoni Armas, 1999. RD. (Fig. 1 a).

Centruroides alayoni Armas, 1999: 122-124, 135. Armas, 2001a: 246. Teruel, 2005: 167-170, 173. Armas, 2006: 3, 4. Perez-Gelabert, 2008: 68. Teruel et al., 2015a: 1, 15.

Centruroides altagraciae Teruel, Armas et Kovařík, 2015. RD. (Fig. 1 b).

Centruroides altagraciae Teruel, Armas y Kovařík, 2015b: 14-24. Armas y Teruel, 2016: 153-154.

Centruroides bani Armas y Marcano Fondeur, 1987. H, RD. (Fig. 1 c).

Centruroides bani Armas y Marcano Fondeur, 1987: 5-12. Fet y Lowe, 2000: 100. Armas, 2001a: 246. Armas, 2002a: 64. Armas, 2006: 3. Perez-Gelabert, 2008: 68. Teruel et al., 2015a: 1, 14-16. Lourenço y Armas, 2015: 226-227.

Centruroides jaragua Armas, 1999. RD. (Fig. 1 d).

Centruroides jaragua Armas, 1999: 124-126. Teruel, 2005: 168-173. Armas, 2006: 3, 5. Perez-Gelabert, 2008: 68. Teruel et al., 2015a: 1, 15.

Centruroides lucidus Teruel, Armas et Kovařík, 2015a. H, RD. (Fig. 1 e).

Centruroides tenuis: Armas et al., 1999: 31 (error de identificación).

Centruroides sp.: Teruel, 2005: 168-169, 171-172.

Centruroides lucidus Teruel et al., 2015a: 1-17. Lourenço y Armas, 2015: 227-228. Teruel et al., 2015b: 20, 23, 24.

Centruroides marcanoi Armas, 1981. RD. (Fig. 1 f).

Centruroides marcanoi Armas, 1981b: 10-12, 21. Armas, 1988a: 55, 93. Wetherbee, 1989: 3, 16. Fet y Lowe, 2000: 112. Teruel, 2000a: 73 (solo ejemplares dominicanos). Armas, 2001a: 246. Teruel, 2005: 168-170, 174. Armas, 2006: 3. Perez-Gelabert, 2008: 68. Teruel et al., 2015a: 1, 15.

Centruroides nitidus (Thorell, 1876). H, RD. (Fig. 1 g).

Centrurus nitidus Thorell, 1876: 152-153. 
Centrurus republicanus Karsch, 1879: 120-121. Moritz y Fisher, 1980: 323. Sinonimizada por Kraepelin (1891: 129).

Centruroides nitidus: Pocock, 1902: 20, 28. Armas, 1981b: 7-8, 12. Armas, 1988a: 56-57, 93, 102. Wetherbee, 1989: 3, 20-21. Fet y Lowe, 2000: 115-116. Armas, 2002a: 61-65 (en parte: excepto registros para La Altagracia). Teruel, 2005: 168-173. Seiter y Teruel, 2014: 127-129. Teruel et al., 2015a: 1, 14, 16. Lourenço y Armas, 2015: 227. Teruel et al., 2015b: 20, 23.

$(\dagger)$ Centruroides beynai Schawaller, 1979: 6-13. Sinonimizada por Armas (1988a: 56-57).

Centruroides nitidus nitidus: Armas y Marcano Fondeur, 1987: 13-14. Fet y Lowe, 2000: 116.

Centruroides nitidus taino Armas y Marcano Fondeur, 1987: 14-16. Fet y Lowe, 2000: 116. Kovařík, 2001: 80. Armas, 2006: 4. Sinonimizada por Armas, 2002a: 61-62, 64.

Centruroides taino: Santiago-Blay, 1993: 6.

Centruroides tenuis: Perez-Gelabert, 2008: 68.

Comentarios. Kraepelin (1891: 129) incluyó a Centrurus tenuis Thorell, 1876, descrita sobre la base de cinco sintipos procedentes de "St. Domingo", "Antillas" y "New York" como un sinónimo más modernos de C. nitidus. Armas (1976a) describió Centruroides zayasi sobre la base de una hembra procedente de Les Cayes, Haití; pero poco después, Armas (1981b) la consideró un sinónimo más moderno de C. nitidus.

Armas y Marcano Fondeur (1987) excluyeron a C. tenuis de la sinonimia de C. nitidus, reconociéndolas como dos especies diferentes. A la vez señalaron que la única especie cuya descripción se correspondía con la de C. tenuis era C. zayasi. Armas (1988a) y Fet y Lowe (2000) incluyeron a C. tenuis y C. zayasi como sinónimos de C. nitidus; pero C. zayasi difiere de C. nitidus lo suficiente como para ser considerada una especie diferente (Armas, 2001a).

El macho de Isla Beata, suroeste de República Dominicana, identificado como C. tenuis por Armas et al. (1999), fue posteriormente reconocido como perteneciente a una especie nueva: C. lucidus (véase Teruel et al., 2015a).

El esclarecimiento de la situación taxonómica de C. tenuis y C. zayasi será próximamente abordado (R. Teruel, L. F. de Armas y F. Kovař́k, en preparación).

Género Isometrus Ehrenberg, 1831. (Fig. 3 b).

Isometrus maculatus (DeGeer, 1778). H, RD (introducida en ambos países). (Fig. 1 h).

Scorpio maculatus DeGeer, 1778: 346-347.

Isometrus maculatus: Pocock, 1893: 376. Armas, 1976b: 3. Armas y Marcano Fondeur, 1987: 1. Armas, 1988a: 60-61. Santiago-Blay, 1993: 6. Armas, 2001a: 246. Perez-Gelabert, 2008: 68. Armas y Teruel, 2016: 153.

Género Microtityus Kjellesvig-Waering, 1966. (Fig. 3 c).

$(\dagger)$ Microtityus ambarensis (Schawaller, 1982).

$(\dagger)$ Tityus ambarensis Schawaller, 1982: 3-14.

(†) Microtityus ambarensis: Armas, 1988b: 1-2. Pérez-Gelabert, 1999: 26. Fet y Lowe, 2000: 183. Santiago-Blay et al., 1990: 115-117. Perez-Gelabert, 2008: 68. Armas y Teruel, 2012: 69-70, 71. 
Microtityus barahona Armas et Teruel, 2012. RD. (Fig. 1 i).

Microtityus consuelo: Armas y Marcano Fondeur, 1992: 7, 16-18, 21 (error de identificación: ejemplares de Playa Azul, Barahona).

Microtityus barahona Armas y Teruel, 2012: 70-75, 78, 87.

Microtityus consuelo Armas et Marcano Fondeur, 1987. RD. (Fig. 1 j).

Microtityus consuelo Armas y Marcano Fondeur, 1987: 16-19. Armas y Marcano Fondeur, 1992: 16-22. Fet y Lowe, 2000: 185. Teruel, 2000b: 34, 35. Armas, 2002b: 100. Armas, 2006: 5. Perez-Gelabert, 2008: 68. Armas y Teruel, 2012: 69, 72-75, 78-80, 82, 87.

Microtityus dominicanensis Santiago-Blay, 1985. RD.

Microtityus dominicanensis Santiago-Blay, 1985: 1-6. Armas, 1988a: 62-63, 93. Armas, 2002b: 99-101. Teruel, 2000b: 34, 35. Fet y Lowe, 2000: 183. Perez-Gelabert, 2008: 68. Armas y Teruel, 2012: 69-71, 76-77, 80, 82-83, 86-87.

Microtityus (Parvabsonus) dominicanensis: Teruel, 2005: 172.

Microtityus iviei Armas, 1999. RD. (Fig. 1 k).

Microtityus iviei Armas, 1999: 104-105. Armas, 2006: 5. Perez-Gelabert, 2008: 68. Armas y Teruel, 2012: 69-70, 72, 75, 81-82, 87.

Microtityus (Parvabsonus) sp.: Teruel, 2005: 168, 170-171, 173-174 (en parte).

Microtityus lantiguai Armas et Marcano Fondeur, 1992. RD. (Fig. 1 1).

Microtityus lantiguai Armas y Marcano Fondeur, 1992: 7-10. Fet y Lowe, 2000: 184. Teruel, 2000b: 34, 35. Armas, 2002a: 61-62, 66-67. Armas, 2002b: 100. Armas, 2006: 5-6. Perez-Gelabert, 2008: 68. Armas y Teruel, 2012: 70-72, 82-83.

Microtityus (Parvabsonus) lantiguai: Teruel, 2005: 166, 168-174.

Microtityus lourencoi Armas et Teruel, 2012. RD.

Microtityus consuelo: Armas y Marcano Fondeur, 1992: 17, 19, 21-22 (error de identificación: ejemplares de Bayahibe).

Microtityus lourencoi Armas y Teruel, 2012: 70-72, 77-78, 87.

Microtityus minimus Kovařík et Teruel, 2014. RD. (Fig. 1 m).

Microtityus minimus Kovařík y Teruel, 2014: 1-7, 10.

Microtityus paucidentatus Armas et Marcano Fondeur, 1992. RD.

Microtityus paucidentatus Armas y Marcano Fondeur, 1992: 11-16. Fet y Lowe, 2000: 184. Teruel, 2000b: 34, 35. Armas, 2002b: 100. Armas, 2006: 6. Perez-Gelabert, 2008: 68. Armas y Teruel, 2012: 69-70, 73, 75-76, 80, 82-83, 85-87. Kovařík y Teruel, 2014: 7.

Microtityus prendinii Armas et Teruel, 2012. RD.

Microtityus consuelo: Armas y Marcano Fondeur, 1992: 21-22 (error de identificación: ejemplares de Samaná).

Microtityus prendinii Armas y Teruel, 2012: 70-73, 83-85, 87. 
Microtityus reini Armas et Teruel, 2012. RD.

Microtityus paucidentatus: Armas y Marcano Fondeur, 1992: 7, 13-14, fig. 4 (error de identificación: ejemplares de Baní, provincia Peravia). Fet y Lowe, 2000: 184 (en parte: ejemplares de la provincia Peravia).

Microtityus reini Armas y Teruel, 2012: 70-71, 74-77, 80, 85, 87. Kovařík y Teruel, 2014: 7.

Microtityus solegladi Armas et Teruel, 2012. RD. (Fig. $1 \mathrm{n}$ ).

Microtityus consuelo: Armas y Marcano Fondeur, 1992: 16-19, 21-22 (error de identificación: ejemplares de Segundo Paso, Neiba).

Microtityus solegladi Armas y Teruel, 2012: 70-74, 83, 87.

Microtityus virginiae Armas, 1999. RD.

Microtityus virginiae Armas, 1999: 102-104. Armas, 2002b: 100. Armas, 2006: 6. Perez-Gelabert, 2008: 68. Armas et Teruel, 2012: 75-77, 80, 86-87.

Microtityus (Parvabsonus) virginiae: Teruel, 2005: 165, 168-169, 172-173.

Microtityus sp. n. RD.

Microtityus dominicanensis: Armas, 2002b: 99-101 (error de identificación: ejemplar de Mata Grande).

Microtityus sp.n.: Armas y Teruel, 2012: 73, 76, 84-87.

Género Rhopalurus Thorell, 1876. (Fig. 3 d).

Rhopalurus abudi Armas et Marcano Fondeur, 1987. RD. (Fig. 1 ñ).

Rhopalurus abudi Armas y Marcano Fondeur, 1987: 19-20. Armas et al., 1999: 30-31. Fet y Lowe, 2000: 217. Lourenço et al., 2000: 141-143. Armas, 2001a: 246. Armas, 2006: 6. Perez-Gelabert, 2008: 68. Prendini et al., 2009: 206-215, 217-220, 222.

Rhopalurus bonettii Armas, 1999. RD. (Fig. 2 a).

Rhopalurus bonettii Armas, 1999: 126-129. Armas, 2006: 6, 10. Prendini et al., 2009: 206-207, 209, 211-213, 215-218, 220, 222-223. Perez-Gelabert, 2008: 68.

Rhopalurus princeps (Karsch, 1879). H, RD. (Fig. 2 b).

Centrurus princeps Karsch, 1879: 121-122. Moritz y Fisher, 1980: 322.

Rhopalurus princeps: Armas, 1981c: 1-7. Lourenço, 1982: 107, 108, 114, 134, 135, 136, 137, 138 (en parte: solamente registros de La Española ). Lourenço, 1984: 169-170. Lourenço, 1986: 135. Armas y Marcano Fondeur, 1987: 20-23. Armas, 1988a: 70-71, 93. Lourenço, 1989: 743-747. Wetherbee, 1989: 3, 22-25. Lourenço y Cloudsley-Thompson, 1995: 424, 426. Fet y Lowe, 2000: 221. Armas, 2001a: 246. Prendini et al., 2009: 206, 207, 210, 211, 212, 213, 216, 218, 219, 220, 222, 223. Perez-Gelabert, 2008: 68. Lourenço y Armas, 2015: 228-229.

Género Tityus C. L. Koch, 1836. (Fig. 3 e).

Tityus abudi Armas, 1999. RD.

Tityus abudi Armas, 1999: 105-107, 108. Armas, 2001a: 246. Armas, 2006: 6, 7. Perez-Gelabert, 2008: 68 . 
Tityus altithronus Armas, 1999. RD.

Tityus altithronus Armas, 1999: 115, 116-117, 118. Armas, 2001a: 246. Armas, 2006: 7. Perez-Gelabert, 2008: 68 .

Tityus anasilviae Armas et Abud Antun, 2004. RD.

Tityus anasilviae Armas y Abud, 2004: 54, 56. Armas, 2006: 7. Perez-Gelabert, 2008: 69. Teruel y Armas, 2006: 140, 141, 147, 148. Armas y Abud Antun, 2015: 136-138.

(†) Tityus azari Lourenço, 2013. RD (en ámbar).

(†) Tityus azari Lourenço, 2013: 1-5.

Tityus bahoruco Teruel et Armas, 2006. RD. (Fig. 2 c).

Tityus bahoruco Teruel y Armas, 2006: 140-143, 147, 148. Perez-Gelabert, 2008: 69.

Tityus bellulus Armas, 1999. RD.

Tityus bellulus Armas, 1999: 112, 113-114, 115. Armas, 2001a: 246. Perez-Gelabert, 2008: 69.

Tityus crassimanus (Thorell, 1876). H, RD. (Fig. 2 d).

Isometrus crassimanus Thorell, 1876: 129-131.

Isometrus antillanus: Thorell, 1876: 134-135. Sinonimizada por Francke y Santiago-Blay (1984: 284).

Phassus crassimanus: Kraepelin, 1891: 111.

Tityus antillanus: Pocock, 1893: 384-385. Francke y Santiago-Blay, 1984: 286, 287, 28. Armas, 1984b: 3-4.

Tityus crassimanus: Kraepelin, 1899: 76. Francke y Santiago-Blay, 1984: 283-288. Armas, 1988a: 75-76, 93. Fet y Lowe, 2000: 240-241. Armas, 2001a: 246. Armas y Abud Antun, 2004: 53, 54, 55, 56. Teruel y Armas, 2006: 141-145, 148. Perez-Gelabert, 2008: 69.

Tityus crassicauda: Armas, 2002a: 66 [lapsus calami].

Tityus ebanoverde Armas, 1999. RD.

Tityus ebanoverde Armas, 1999: 118, 119. Armas, 2001a: 246, 248. Armas, 2006: 7, 11. Perez-Gelabert, 2008: 69.

Tityus elii Armas et Marcano Fondeur, 1992. RD. (Fig. 2 e).

Tityus elii Armas y Marcano Fondeur, 1992: 22, 23-25, 26. Armas, 2001a: 246. Armas, 2006: 7-8.

Perez-Gelabert, 2008: 69.

Caribetityus elii: Lourenço, 1999: 136-137. Rouaud et al., 2002: 87-89. Kovařík, 2001: 83.

(†) Tityus geratus Santiago-Blay y Poinar, 1988. RD (en ámbar).

(†) Tityus geratus Santiago-Blay y Poinar, 1988: 345-354. Fet y Lowe, 2000: 247. Perez-Gelabert, 1999: 26. Perez-Gelabert, 2008: 69.

(†) Tityus (Brazilotityus) hartkorni Lourenço, 2009. RD (en ámbar).

(†) Tityus (Brazilotityus) hartkorni Lourenço, 2009: 1-4.

Tityus kindli Kovař́k et Teruel, 2014. RD. (Fig. 2 f). 
Tityus kindli Kovařík y Teruel, 2014: 10, 12, 14, 15, 16.

Tityus neibae Armas, 1999. RD.

Tityus neibae Armas, 1999: 110, 111, 112. Armas, 2001a: 246. Armas, 2006: 8. Perez-Gelabert, 2008: 69.

Tityus ottenwalderi Armas, 1999. RD.

Tityus ottenwalderi Armas, 1999: 108, 109,110. Armas, 2001a: 246. Teruel y Armas, 2006: 140, 146, 147, 148. Armas, 2006: 8-9. Perez-Gelabert, 2008: 69.

Tityus portoplatensis Armas et Marcano Fondeur, 1992. RD.

Tityus portoplatensis Armas y Marcano Fondeur, 1992: 30, 31-32, 33. Armas, 2001a: 246. Armas, 2006: 9. Perez-Gelabert, 2008: 69.

Tityus quisqueyanus Armas, 1982. RD. (Fig. 2 g).

Tityus quisqueyanus Armas, 1982b: 13-18. Armas, 1987: 1-17. Wetherbee, 1989: 3, 26. Armas, 2001a: 246. Armas, 2006: 9. Kovař́́k y Teruel, 2014: 14, 16.

Caribetityus quisqueyanus: Lourenço, 1999: 138, 140.

Tityus septentrionalis Armas et Abud Antun, 2004. RD.

Tityus septentrionalis Armas y Abud Antun, 2004: 56, 58, 59. Armas, 2005: 2-3. Armas, 2006: 9. Perez-Gelabert, 2008: 69.

FAMilia HoRmuridae LAURIE, 1896

Género Opisthacanthus Peters, 1861. (Fig. 3 f).

Opisthacanthus lepturus (Palisot de Beauvois, 1805). H, RD. (Fig. 2 h).

Scorpio lepturus Palisot de Beauvois, 1805: 191.

Opisthacanthus kingbergii laevicauda Thorell, 1876: 247-249. Sinonimizada por Lourenço (1979: 29), pero véase Lourenço (1995).

Opisthacanthus elatus: Pocock, 1893: 398 (error de identificación). Kraepelin, 1899: 148 (en parte). Kraepelin, 1901:272 (error de identificación).

Opisthacanthus elatus laevicauda: Kraepelin, 1899: 148-149.

Opisthacanthus lepturus: Lourenço, 1981: 345 (en parte). Armas, 1982: 3, 5. Lourenço, 1988: 17-18. Armas y Marcano Fondeur, 1987:2. Armas, 1988a: 129. Lourenço, 1992: 48, 49. Rudloff, 1994a: 7. Rudloff, 1994b: 21-22. Lourenço, 1995: 79. Fet, 2000: 402. Armas, 2001a: 246.

Opisthacanthus laevicauda: Armas y Marcano Fondeur, 1992: 2-7. Teruel, 2005: 165-166, 168.

FAMilia Scorpionidae Latreille, 1802

Subfamilia Diplocentrinae Karsch, 1880.

Género Cazierius Francke, 1978. (Fig. 3 g).

Cazierius cicero (Armas et Marcano Fondeur, 1987). RD. (Fig. 2 i). 
Heteronebo cicero Armas y Marcano Fondeur, 1987: 3-5. Sissom y Fet, 2000: 346. Armas, 2006: 11.

Cazierius cicero: Kovař́k, 1998: 130. Armas et al., 1999: 30. Armas, 2001: 246. Perez-Gelabert, 2008: 69.

Kovařík y Teruel, 2014: 24-25.

Cazierius neibae Kovařík et Teruel, 2014. H, RD. (Fig. 2 j).

Cazierius neibae Kovařík y Teruel, 2014:16-25. Lourenço y Armas, 2015: 230.

Cazierius politus (Pocock, 1898). RD. (Fig. 2 k).

Diplocentrus politus Pocock, 1898: 390.

Cazierius politus: Francke, 1978: 28-30, 56. Armas, 1981a: 10-12; 1982: 5. Armas y Marcano

Fondeur, 1987: 2-3. Armas, 1988a: 22. Rudloff, 1994a: 7; 1994b: 24. Kovař́́k, 1998: 130. Sissom y Fet, 2000: 333. Armas, 2001a: 246. Teruel, 2005: 166, 170. Kovař́k y Teruel, 2014: 7, 24, 25.

Género Heteronebo Pocock, 1899. (Fig. 3 h).

Heteronebo barahonae Teruel, Armas et Kovařík, 2015. RD. (Fig. 2 1).

Heteronebo barahonae Teruel et al., 2015b: 24-32.

Heteronebo dominicus Armas, 1981. RD. (Fig. 2 m).

Heteronebo dominicus Armas, 1981a: 5-9. Armas, 1988a: 31. Rudloff, 1994a: 7. Kovařík, 1998: 131. Sissom y Fet, 2000: 346. Teruel, 2005: 166.

Cazierius dominicus: Armas, 1999: 132. Armas, 2001a: 246.

Heteronebo monticola (Armas, 1999). RD. (Fig. 2 n).

Cazierius monticola Armas, 1999: 131-133. Armas, 2001a: 246. Armas, 2006: 10.

Heteronebo monticola: Teruel, 2005: 167. Teruel et al., 2015b: 32.

Heteronebo oviedo (Armas, 1999). H, RD. (Fig. 2 ñ).

Cazierius oviedo Armas, 1999: 133-134. Armas, 2001a: 246. Armas, 2006: 10.

Heteronebo oviedo: Teruel, 2005: 167. Lourenço y Armas, 2015: 230.

Heteronebo pumilus Armas, 1981. H.

Heteronebo pumilus Armas, 1981a: 2-5, 6. Armas, 1984a: 5. Sissom y Fet, 2000: 346. Armas, 2006: 12. Teruel et al., 2015b: 32. 

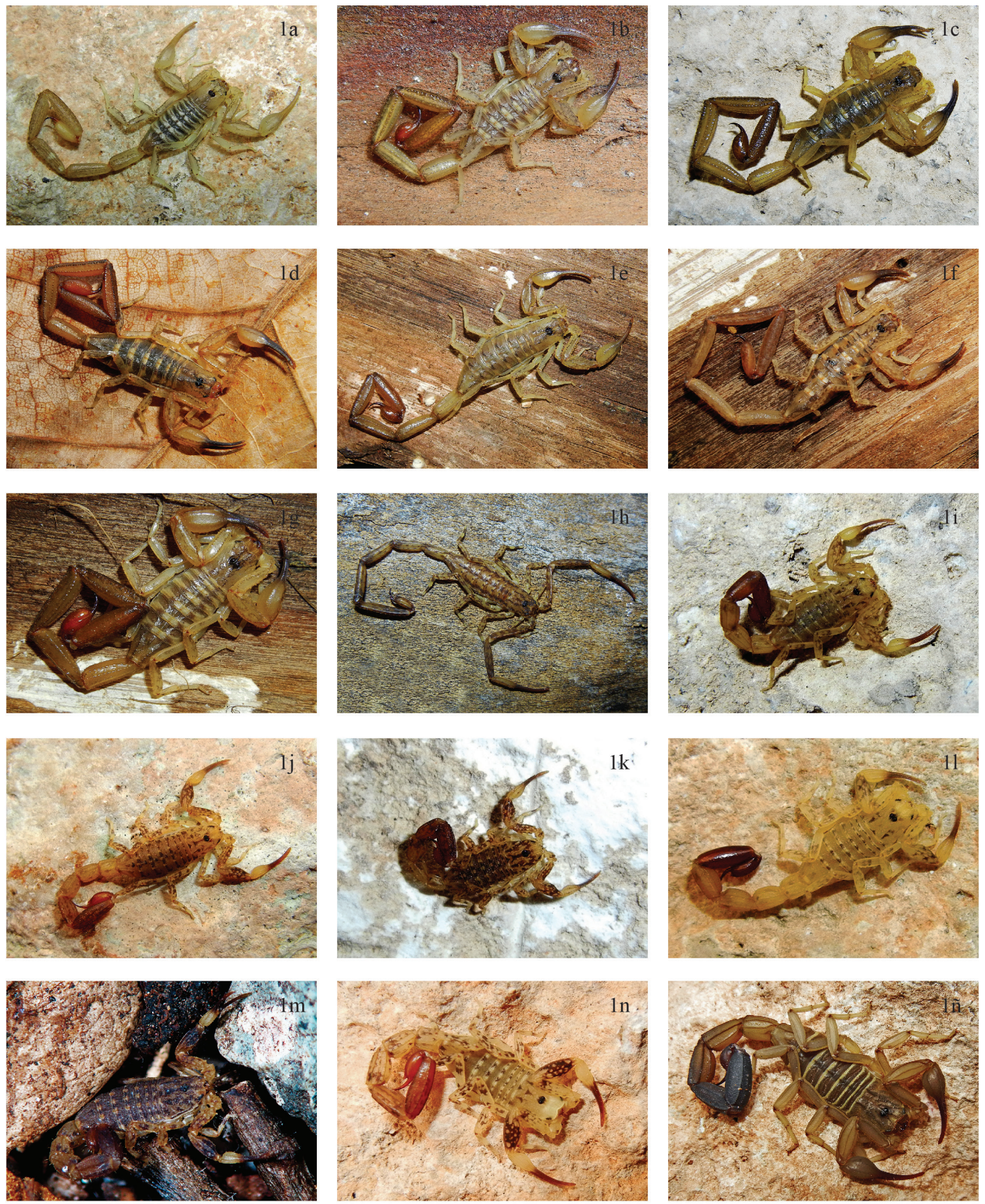

Figura 1. Escorpiones de La Española, fotografiados vivos en su hábitat natural. a) Centruroides alayoni, macho en la carretera Cabo Rojo-El Aceitillar, Pedernales; b) Centruroides altagraciae, macho paratipo de Boca de Yuma, La Altagracia; c) Centruroides bani, macho en El Número, Azua; d) Centruroides jaragua, macho de Maniel Viejo, Barahona; e) Centruroides lucidus, macho paratipo de Los Tres Charcos, Pedernales; f) Centruroides marcanoi, macho topotipo; g) Centruroides nitidus, macho de Río Mulito, Pedernales; h) Isometrus maculatus, macho de Sabana, Maisí, Cuba; i) Microtityus barahona, macho topotipo; j) Microtityus consuelo, macho topotipo; k) Microtityus iviei, macho de Sabana de Sansón, Pedernales; 1) Microtityus lantiguai, macho en la carretera Cabo Rojo-El Aceitillar, Pedernales; m) Microtityus minimus, macho holotipo; n) Microtityus solegladi, macho de Segundo Paso, Bahoruco; ñ) Rhopalurus abudi, macho en Boca de Yuma, La Altagracia. 

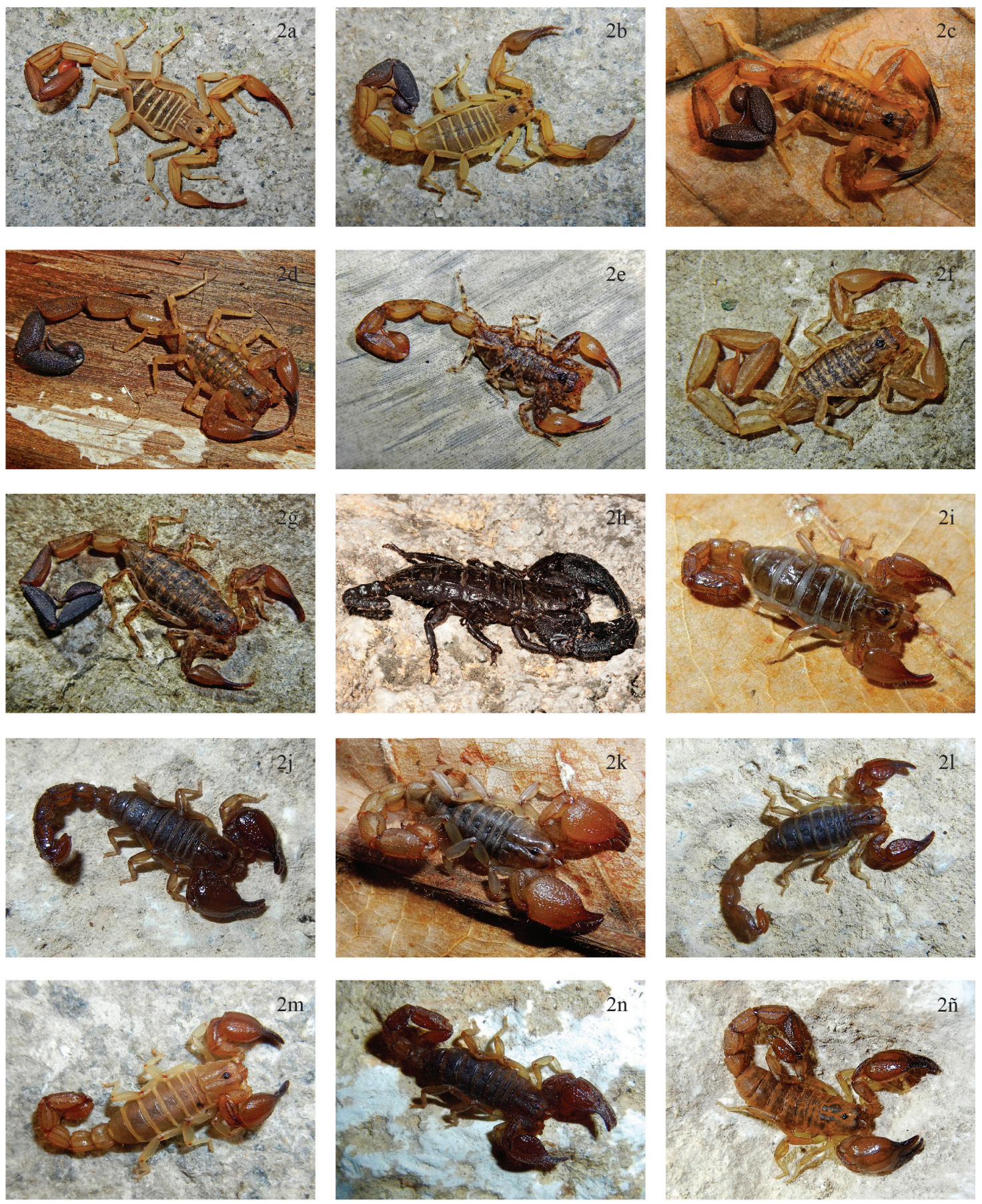

Figura 2. Escorpiones de La Española, fotografiados vivos en su hábitat natural. a) Rhopalurus bonettii, macho en la carretera Cabo Rojo-El Aceitillar, Pedernales; b) Rhopalurus princeps, macho de Puerto Escondido, Independencia; c) Tityus bahoruco, macho en la carretera Cabo Rojo-El Aceitillar, Pedernales; d) Tityus crassimanus, macho de Río Mulito, Pedernales; e) Tityus elii, macho de Casabito, La Vega; f) Tityus kindli, macho holotipo; g) Tityus quisqueyanus, macho topotipo; h) Opisthacanthus lepturus, hembra de Los Haitises, Hato Mayor (foto cortesía del Padre Alejandro Sánchez); i) Cazierius cicero, hembra de Bayahibe, La Altagracia; j) Cazierius neibae, macho holotipo; k) Cazierius politus, macho de El Número, Azua; 1) Heteronebo barahonae, macho holotipo; m) Heteronebo dominicus, macho en Las Mercedes, Pedernales; n) Heteronebo monticola, macho en la carretera Cabo Rojo-El Aceitillar, Pedernales; ñ) Heteronebo oviedo, macho topotipo. 

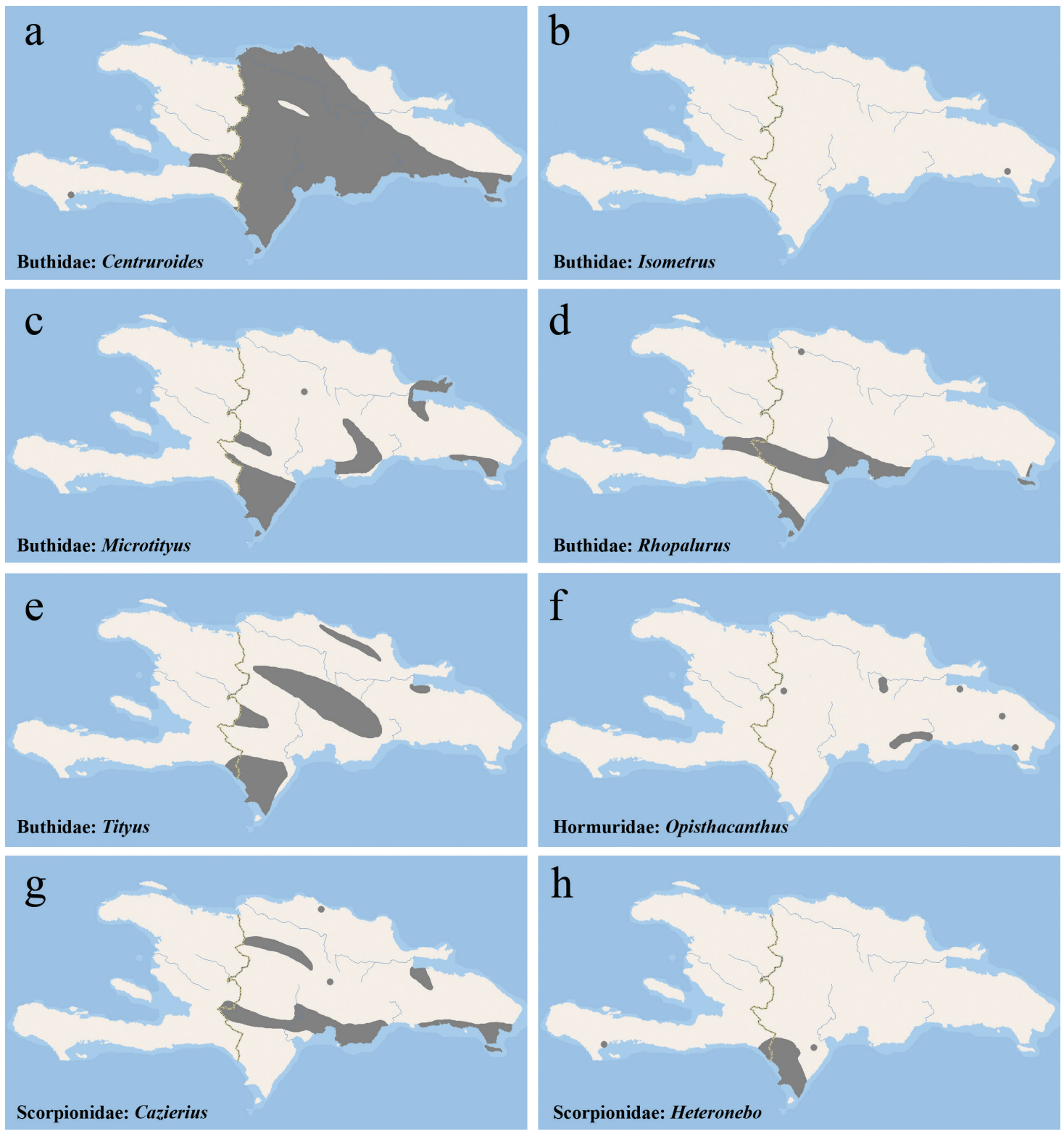

Figura 3. Distribución geográfica actualizada de los ocho géneros de escorpiones de La Española . a) Centruroides; b) Isometrus; c) Microtityus; d) Rhopalurus; e) Tityus; f) Opisthacanthus; g) Cazierius; h) Heteronebo. 


\section{DISCUSIÓN}

Las diferencias en la composición taxonómica de las respectivas escorpiofaunas de Haití y República Dominicana son el reflejo de varios factores, entre los que son determinantes la menor extensión territorial del primero de estos dos países, la fuerte deforestación a que ha estado sometido el mismo durante los últimos siglos y la poca atención que han recibido estos arácnidos en dicho territorio. Lourenço y Armas (2015) registraron la presencia en Haití de cuatro especies previamente descritas de localidades dominicanas, por lo que es de esperar que futuras investigaciones en áreas haitianas próximas a la frontera con República Dominicana proporcionen el descubrimiento de otros casos similares. Especies como Cazierius politus, Heteronebo dominicus, H. monticola, Centruroides alayoni, C. jaragua, C. marcanoi, Microtityus barahona, M. iviei, M. lantiguai, M. solegladi, M. virginiae, Rhopalurus bonettii, Tityus bahoruco y T. neibae también pudieran estar presentes en Haití, pues han sido halladas a escasos kilómetros de la frontera con este país, incluso en el mismo continuo de vegetación y altitud.

Por otra parte, tampoco puede afirmarse que la escorpiofauna dominicana haya sido exhaustivamente investigada. Según datos inéditos de los autores, alrededor de una decena de especies serán descritas o sinonimizadas en los próximos años, en tanto la información taxonómica y biológica sobre otras se enriquecerá de modo sustancial.

Entre las tareas que habrán de emprenderse en un futuro inmediato están: (1) el esclarecimiento de la identidad taxonómica precisa de Centruroides tenuis, C. zayasi, Tityus anasilviae, T. bahoruco y T. ebanoverde; (2) la revisión del género Cazierius en la isla; (3) el completamiento de la información taxonómica de varias especies descritas de un único ejemplar o de especímenes de uno solo de los sexos. Otras tareas a más largo plazo deberán abordar el análisis de las relaciones biogeográficas y filogenéticas de esta fauna, temas sobre los que los análisis hasta ahora realizados son muy superficiales o insuficientes. Y por supuesto, habrá que dedicarle un más decidido esfuerzo al completamiento de la información sobre la escorpiofauna de la parte haitiana.

\section{AGRADECIMIENTOS}

Durante muchos años, numerosos amigos y colegas nos han proporcionado bibliografía, a veces de difícil acceso: Victor Fet (Marshall University, West Virginia, EE. UU.), Frantíšek Kovař́k (Praga, República Checa), Oscar F. Francke (Universidad Nacional Autónoma de México, México), Wilson R. Lourenço (Museum National d'Histoire Naturelle, Paris) y Norman I. Platnick (American Museum of Natural History, Nueva York, EE. UU.). También ha sido invaluable la donación o envío de especímenes y el apoyo logístico durante el trabajo de campo por parte de otros como el propio F. Kovařík, Pavel Kindl (Praga, República Checa) y Abraham J. Abud Antun (Junta Agroempresarial Dominicana, Santo Domingo, República Dominicana). Eugenio de Jesús Marcano Fondeur (Universidad Autónoma de Santo Domingo, Santo Domingo, República Dominicana) y Julio Cicero SJ (Politécnico Ignacio Loyola, República Dominicana), ambos ya fallecidos, hicieron en su momento importantes aportes de los que se nutre esta publicación, a ellos nuestro homenaje. 


\section{LITERATURA CITADA}

Armas, L. F. de. 1976a. Escorpiones del archipiélago cubano. V. Nuevas especies de Centruroides (Scorpionida: Buthidae). Poeyana, 146: 1-55.

Armas, L. F. de. 1976b. Notas sobre la distribución geográfica de Isometrus maculatus (De Geer) (Scorpionida: Buthidae) en las Antillas. Miscelánea Zoológica (La Habana), 5: 3-4.

Armas, L. F. de. 1981a. Primeros hallazgos de la familia Diplocentridae (Arachnida: Scorpionida) en La Española . Poeyana, 213:1-12.

Armas, L. F. de. 1981b. El género Centruroides Marx, 1889 (Scorpiones: Buthidae) en Bahamas y República Dominicana. Poeyana, 223:1-21.

Armas, L. F. de. 1981c. Redescripción de Rhopalurus princeps (Karsch, 1879) (Scorpionida: Buthidae. Poeyana, 227: 1-7.

Armas, L. F. de. 1982a. Distribución y biogeografía del género Rhopalurus Thorell (Scorpiones: Buthidae) en Cuba. Miscelánea Zoológica (La Habana), 17: 4.

Armas, L. F. de. 1982b. Adiciones a las escorpiofaunas (Arachnida: Scorpiones) de Puerto Rico y República Dominicana. Poeyana, 237: 1-25.

Armas, L. F. de. 1984a. Tipos de Arachnida depositados en el Instituto de Zoología de la Academia de Ciencias de Cuba. I. Amblypygi, Opiliones, Ricinulei, Scorpiones, Schizomida, y Uropygi. Poeyana, 284: 1-11.

Armas, L. F. de. 1984b. Primera localidad precisa para Tityus antillanus (Thorell, 1877) (Scorpiones: Buthidae). Miscelánea Zoológica (La Habana), 21: 3-4.

Armas, L. F. de. 1986. El alacrán. Gente Nueva, La Habana. 51 pp.

Armas, L. F. de. 1987. Morfometría de Tityus quisqueyanus Armas (Scorpiones: Buthidae) con notas sobre su historia natural. Poeyana, 338: 1-17.

Armas, L. F. de. 1988a. Sinopsis de los escorpiones antillanos. Científico-Técnica, La Habana, $102 \mathrm{pp}$.

Armas, L. F. de. 1988b. Situación taxonómica de Tityus ambarensis (Scorpiones: Buthidae) escorpión fósil de República Dominicana. Garciana, Holguín, 11: 1-2.

Armas, L. F. de. 1999. Quince nuevos alacranes de La Española y Navassa, Antillas Mayores (Arachnida: Scorpiones). Avicennia, 10-11: 101-136.

Armas, L. F. de. 2001a. Scorpions of the Greater Antilles, with the description of a new troglobitic species (Scorpiones: Diplocentridae). Pp. 245-253 en Scorpions 2001. In Memoriam Gary A. Polis (V. Fet y P. A. Selden, eds.). Burnham Beeches, Bucks: British Arachnological Society.

Armas, L. F. de. 2001b. Frogs and lizards as prey of some Greater Antillean arachnids. Revista Ibérica de Aracnología, 3: 87-88. 
Armas, L. F. de. 2002a. Alacranes de República Dominicana. Centruroides nitidus (Thorell, 1876) y Microtityus lantiguai Armas y Marcano Fondeur, 1992 (Scorpiones: Buthidae). Revista Ibérica de Aracnología, 5: 61-66.

Armas, L. F. de. 2002b. Redescubrimiento del alacrán Microtityus dominicanensis Santiago-Blay (Scorpiones: Buthidae) de República Dominicana. Revista Ibérica de Aracnología, 5: 99-101.

Armas, L. F. de. 2005. Antillean scorpions deposited at the Montana State University (Arachnida: Scorpiones). Euscorpius, 18: 1-4.

Armas, L. F. de. 2006. Name-bearing types of scorpions deposited at the Institute of Ecology and Systematics, Havana (Arachnida: Scorpiones). Euscorpius, 33: 1-14.

Armas, L. F. de y A. [J.] Abud [Antun]. 1992. Depredación de vertebrados por escorpiones (Scorpiones: Buthidae) de República Dominicana. Pp. 5-6 en Comunicaciones breves de Zoología, Editorial Academia, La Habana.

Armas, L. F. de y A. J. Abud Antun. 2000. El alacrán en la cultura de República Dominicana. Revista Ibérica de Aracnología, 1: 77-79.

Armas, L, F. de y A. J. Abud Antun. 2004. Adiciones al género Tityus C. L. Koch, 1836 en República Dominicana, con la descripción de dos especies nuevas (Scorpiones: Buthidae). Revista Ibérica de Aracnología, 10: 53-64.

Armas, L. F. de y A. J. Abud Antun. 2015. Descripción de la hembra de Tityus anasilviae Armas y Abud Antun, 2004 (Scorpiones: Buthidae) de República Dominicana. Revista Ibérica de Aracnología, 27: 136-138.

Armas, L. F. de y E. J. Marcano Fondeur. 1987. Nuevos escorpiones (Arachnida: Scorpiones) de República Dominicana. Poeyana, 356: 1-24.

Armas, L. F. de y E. J. Marcano Fondeur. 1988. Adiciones a la aracnofauna de República Dominicana (Arachnida: Scorpiones, Palpigradi, Acarina). I Simposio de Zoología, La Habana, 14-17 de junio de 1988. Resúmenes, p. 129.

Armas, L. F. de y E. J. Marcano Fondeur. 1992. Nuevos alacranes de República Dominicana (Arachnida: Scorpiones). Poeyana, 420: 1-36.

Armas, L. F. de, J. A. Ottenwalder y K. A. Guerrero. 1999. Alacranes de las islas Saona, Beata y Catalina, República Dominicana (Arachnida: Scorpiones). Cocuyo (La Habana), 8: 30-32.

Armas, L. F. de y D. Prieto Trueba. 2003. Primer registro de ácaros parásitos de amblipígidos (Arachnida: Amblypygi). Revista Ibérica de Aracnología, 7: 133-134.

Armas, L. F. de y R. Teruel, 2012. Revisión del género Microtityus Kjellesvig-Waering, 1966 (Scorpiones: Buthidae) en República Dominicana. Revista Ibérica de Aracnología, 21: 69-88.

Armas, L. F. de y R. Teruel. 2016. Ampliación del área de distribución conocida de Centruroides altagraciae (Scorpiones: Buthidae). Revista Ibérica de Aracnología, 28: 153-154.

De Geer, C. 1778. Mémoires pour servir à l'histoire des insectes. Stockholm: Imprimerie Pierre Hesselberg, 7, $950 \mathrm{pp}$. 
Fet, V. 2000. Family Ischnuridae Simon, 1879. Pp. 383-408 en Catalog of the scorpions of the world (1758-1998) (V. Fet et al., eds.). New York, The New York Entomological Society.

Fet, V. y G. Lowe. 2000. Family Buthidae C. L. Koch, 1837. Pp. 54-286.en Catalog of the scorpions of the world (1758-1998) (V. Fet et al., eds.). New York, The New York Entomological Society.

Fet, V., W. D. Sissom, G. Lowe y M. E. Braunwalder (ed.) 2000. Catalog of the scorpions of the world (1758-1998) New York, The New York Entomological Society.

Fet, V. y M. E. Soleglad. 2005. Contributions to scorpion systematics. I. On recent changes in high-level taxonomy. Euscorpius, 31: 1-13.

Francke, O. F. 1978. Systematic revision of diplocentrid scorpions (Diplocentridae) from circum-Caribbean lands. Special Publication of the Museum, Texas Tech University, 14: $1-92$.

Francke, O. F. y J. A. Santiago-Blay. 1984. Redescription of Tityus crassimanus (Thorell, 1877), and its junior synonym Tityus antillanus (Thorell, 1877) (Scorpiones, Buthidae). Journal of Arachnology, 12: 283-290.

Karsch, F. 1879. Scorpionologische beiträge. II. Mitteilungen des Münchener Entomologischen Vereins, 3: 97-136.

Kovař́k, F. 1998. Štíri [Scorpiones] «Madagaskar», Jihlava, República Checa. 176 pp. [en checo].

Kovařík, F. 2001. Catalog of the scorpions of the world (1758-1998) by V. Fet, W. D. Sissom, G. Lowe \& M. Braunwalder (New York Entomological Society, 2000: 690 pp.). Discussion and supplement for 1999 and part of 2000. Serket, 7 (3): 78-93.

Kovař́k, F. y R. Teruel. 2014. Three new scorpion species from the Dominican Republic, Greater Antilles (Scorpiones: Buthidae, Scorpionidae). Euscorpius, 187: 1-27.

Kraepelin, K. 1891. Revision der skorpione. I. Die familie der Androctonidae. Jahrbuch der Hamburgischen Wissenschaftlichen Anstalten, 8: 1-139.

Kraepelin, K. 1899. Scorpiones und Pedipalpi. En Das Tierreichs, Friedländer Verlag, Berlín, 8: $1-265$.

Kraepelin, K. 1901. Catalogue des scorpions des collections du Muséum d'histoire naturelle de Paris. Bulletin du Muséum National d'Histoire Naturelle, Paris, 7: 265-273.

Lourenço, W. R. 1979. Liste des espèces et sous-espèces appartenant au genre Opisthacanthus Peters, 1861 (Scorpiones, Scorpionidae). Revista Nordestina da Biologia, 2 (1-2): 27-36.

Lourenço, W. R. 1981. Sur la distribution géographique et l'écologie de Opisthacanthus cayaporum Vellard, 1932 (Scorpiones, Scorpionidae). Revista Brasileira de Biologia, 41 (2): 343-349.

Lourenço, W. R. 1982. Révision du genre Rhopalurus Thorell, 1876 (Scorpiones, Buthidae). Revue de Arachnologie, 4: 107-141. 
Lourenço, W. R. 1984. Complementary notes on the genus Rhopalurus for the Caribbean (Scorpiones, Buthidae). Revista Brasileira de Biologia, 44 (2): 160-170.

Lourenço, W. R. 1986. Biogéographie et phylogénie des scorpions du genre Rhopalurus Thorell, 1876 (Scorpiones, Buthidae). Mémoires de la Société Royale Belge d'Entomologie, 33: 129-137.

Lourenço, W. R. 1988. Considérations biogéographiques, écologiques et évolutives sur les espèces néotropicales d'Opisthacanthus Peters, 1861 (Scorpiones, Ischnuridae). Studies on Neotropical Fauna and Environmental, 23 (1): 41-53.

Lourenço, W. R. 1989. Le développement postembryonnaire de Rhopalurus princeps (Karsch, 1879) (Scorpiones, Buthidae). Revista Brasileira de Biologia, 49 (3): 743-747.

Lourenço, W. R. 1992. Les peuplements des scorpions des Antilles; facteurs historiques et écologiques en association avec les stratégies biodémographiques. Studies on Neotropical Fauna and Environmental, 27 (1): 43-62.

Lourenço, W. R. 1995. Nouvelles considérations sur la classification et la biogéographie des Opisthacanthus neotropicaux (Scorpiones, Ischnuridae). Biogeographica, 71 (2): 75-82.

Lourenço, W. R. 1999. Origines et affinités des scorpions des Grandes Antilles: le cas particulier des éléments de la famille des Buthidae. Biogeographica, 75 (3): 131-144.

Lourenço, W. R. 2009. A new species of Tityus C. L. Koch, 1836 (subgenus Brazilotityus Lourenço, 2006) from the Dominican amber (Scorpiones: Buthidae). Euscorpius, 83: 1-5.

Lourenço, W. R. 2013. A new species of Tityus C. L. Koch, 1836 (Scorpiones: Buthidae) from Dominican amber. Euscorpius, 156: 1-5.

Lourenco, W. R. y L. F. de Armas. 2015. New records of scorpions from Haiti (Scorpiones: Buthidae, Diplocentridae). Entomologische Mitteilungen aus dem Zoologischen Museum Hamburg, 17 (194): 225-232.

Lourenço, W. R. y J. L. Cloudsley-Thompson. 1995. Stridulatory apparatus and the evolutionary significance of sound production in Rhopalurus species (Scorpiones: Buthidae). Journal of Arid Environment, 31: 423-429.

Lourenço, W. R., D. Huber y J. L. Cloudsley-Thompson. 1999. Notes on the postembryonic development of two species of Microtityus Kjellesvig-Waering from Trinidad and Dominican Republic (Scorpiones, Buthidae). Acta Biologica Paranense, Curitiba, 28 (1-4): 1-9.

Lourenço, W. R., D. Huber y J. L. Cloudsley-Thompson. 2000. Description of the stridulatory apparatus in some species of the genus Rhopalurus Thorell (Scorpiones: Buthidae). Ekológia (Bratislava), 19 (suppl. 3): 141-144.

Moritz, M. y S.-C. Fischer. 1980. Die typen der arachniden-sammlung des Zoologischen Museums Berlin. Mitteilungen aus dem Zoologischen Museum in Berlin, 56 (2): 309-326.

Palisot de Beauvois, A.-M.-F.-J. 1805. Insectes recueilles en Afrique et en Amérique, dans les royaumes d'Oware et de Benin, à Saint-Domingue et dans les États-Unis, pendant les années 1786-1797. Paris, Fain et Compagnie: 189-191 (escorpiones). 
Pérez-Gelabert, D. E. 1999. Catálogo sistemático y bibliografía de la biota fósil en ámbar de la República Dominicana. Hispaniolana, nueva serie, 1: 1-65.

Perez-Gelabert, D. E. 2008. Arthropods of Hispaniola (Dominican Republic and Haiti): A checklist and bibliography. Zootaxa, 1831: 1-530.

Pocock, R. I. 1893. Contribution to our knowledge of the arthropod fauna of the West Indies. Part I. Scorpiones and Pedipalpi; with supplementary note upon the freshwater Decapoda of St. Vincent. Journal of the Linnean Society of Zoology, 24 (155): 374-404.

Pocock, R. I. 1898. Descriptions of some new scorpions from Central and South America. Annals and Magazine of Natural History, 7 (1): 384-394.

Pocock, R. I. 1902. Arachnida. Scorpiones, Pedipalpi, and Solifugae. En Biologia Centrali-Americana, Taylor y Francis, Londres, 71 pp. + 12 láminas.

Prendini, L., L. A. Esposito, J. C. Huff y E. S. Volschenk. 2009. Redescription of Rhopalurus abudi (Scorpiones, Buthidae), with first description of the male and first record from mainland Hispaniola. Journal of Arachnology, 37: 206-224.

Prendini, L. y W. C. Wheeler. 2005. Scorpion higher phylogeny and classification, taxonomic anarchy, and standards for peer review in online publishing. Cladistics, 21: 446-494.

Roewer, C. F. 1943. Über eine neuerworbene Sammlung von Skorpionen des Natur-Museums Senckenberg. Senckenbergiana, 26 (4): 205-244.

Rouaud, Ch., D. Huber y W. Lourenço. 2002. Life history of Caribetityus elii (Armas y Marcano Fondeur, 1992) from the Dominican Republic (Scorpiones, Buthidae). Pp. 87-90 en European Arachnology 2000 (S. Toft y N. Scharff, eds.) (Proceeding of the $19^{\text {th }}$ European Colloquium of Arachnology, Århus 17-22 July, 2000).

Rudloff, J.-P. 1994a. Die skorpiosfauna der Antillen (Arachnida: Scorpiones). Teil I. Arthropoda, 2 (1): 3-12.

Rudloff, J.-P. 1994b. Die skorpiosfauna der Antillen (Arachnida: Skorpiones). Teil II. Arthropoda, 2 (2): 17-30.

Santiago-Blay, J. A. 1985. Microtityus dominicanensis: A new scorpion from the Dominican Republic, West Indies (Scorpiones: Buthidae). Entomological News, 96 (1): 1-6.

Santiago-Blay, J. A. 1990. Systematics and some aspects of the biology of the scorpions (Arachnida: Scorpiones) of Hispaniola (Dominican Republic and Haiti), West Indies. Ph. D. thesis, University of California, Berkeley. [Consultado el resumen solamente].

Santiago-Blay, J. A. 1993. The Scorpions of Puerto Rico and Hispaniola. American Arachnology, 47: 6-7.

Santiago-Blay, J. A. y G. O. Poinar, Jr. 1988. A fossil scorpion Tityus geratus new species (Scorpiones: Buthidae) from Dominican amber. Historical Biology, 1: 345-354.

Santiago-Blay, J. A y P. R. Craig. 1998. The order Schizomida (Arachnida) in the amber fossil record. Presentación Oral en el Congreso Mundial sobre Inclusiones en Ámbar, Alava, España, 20-23 de octubre de 1998, Resúmenes, p. 93. 
Santiago-Blay, J. A., W. Schawaller y G. O. Poinar, Jr. 1990. A new specimen of Microtityus ambarensis (Scorpiones, Buthidae), fossil from Hispaniola: evidence of taxonomic status and possible biogeographic implications. Journal of Arachnology, 18: 115-117.

Schawaller, W. 1979. Ersnachweis eines skorpions in Dominikanischem bernstein (Stuttarter Bernsteinsammlung: Arachnida, Scorpionida). Stuttgarter Beitrage Naturkunde, serie B (Geologie und Palaöntologie), 45: 1-15.

Schawaller, W. 1982. Zwei weitere skorpione in Dominikanischem bernstein (Stuttarter Bernsteinsammlung: Arachnida, Scorpionida). Stuttgarter Beitrage Naturkunde (Geol. Paleontol.) serie B (Geologie und Palaöntologie), 82: 1-14.

Seiter, M. y R. Teruel. 2014. Two new cases of metasomal duplication in scorpions, with notes on their reproductive biology (Scorpiones: Buthidae). Revista Ibérica de Aracnología, 24: $127-129$.

Sissom, W. D. y V. Fet. 2000. Family Diplocentridae Karsch, 1880. Pp. 329-354 en Catalog of the scorpions of the world (1758-1998) (V. Fet et al., eds.). New York, The New York Entomological Society.

Teruel, R. 2000a. Presencia de Centruroides marcanoi Armas, 1981 (Scorpiones: Buthidae) en Cuba. Boletín de la Sociedad Entomológica Aragonesa, 27: 73.

Teruel, R. 2000b. Una nueva especie de Microtityus Kjellesvig-Waering, 1968 (Scorpiones: Buthidae) de Cuba oriental. Revista Ibérica de Aracnología, 1: 31-35.

Teruel, R. 2005. Nuevos datos sobre la taxonomía, distribución geográfica y ecología de los escorpiones de la República Dominicana (Scorpiones: Liochelidae, Scorpionidae, Buthidae). Boletín de la Sociedad Entomológica Aragonesa, 36: 165-176.

Teruel, R. y L. F. de Armas. 2006. Revisión del grupo "Tityus crassimanus" (Scorpiones: Buthidae), con la descripción de una nueva especie de la República Dominicana. Boletín de la Sociedad Entomológica Aragonesa, 39: 139-149.

Teruel, R. y L. F. de Armas. 2012. Redescripción de Rhopalurus junceus (Herbst 1800) (Scorpiones: Buthidae). Boletín de la Sociedad Entomológica Aragonesa, 50: 153-174.

Teruel, R., L. F. de Armas y F. Kovařík. 2015a. A new species of Centruroides Marx, 1890 (Scorpiones: Buthidae) from southern Hispaniola, Greater Antilles. Euscorpius, 198: 1-18.

Teruel, R., L. F. de Armas y F. Kovař́ik. 2015b. Two new species of scorpions (Scorpiones: Buthidae, Scorpionidae) from Dominican Republic, Greater Antilles. Revista Ibérica de Aracnología, 27: 13-33.

Thorell, T. 1876. Étude scorpiologiques. Atti della Società Italiana di Scienze Naturali, 19: $75-272$.

Wetherbee, D. K. 1989. A brief guide to the partly-known fauna of alacranes (Scorpionida) of Hispaniola. Shelburne, Massachusetts. 27 pp.

[Recibido: 14 de julio, 2016. Aceptado para publicación: 27 de septiembre, 2016] 